\title{
HER2 mutations in Chinese patients with non-small cell lung cancer
}

\author{
Zhengbo Song ${ }^{1,2}$, Xinmin Yu ${ }^{1,2}$, Zhiyong Shi ${ }^{1,2}$, Jun Zhao ${ }^{1,2}$ and Yiping Zhang ${ }^{1,2}$ \\ ${ }^{1}$ Department of Medical Oncology , Zhejiang Cancer Hospital, Hangzhou, China \\ ${ }^{2}$ Key Laboratory Diagnosis and Treatment Technology on Thoracic Oncology, Hangzhou, China \\ Correspondence to: Yiping Zhang, email: zjzlyy16@163.com \\ Keywords: non-small cell lung cancer, HER2 mutation, prevalence, genetic variability, treatment \\ Received: November 14,2015 Accepted: July 27, $2016 \quad$ Published: August 16, 2016
}

\section{ABSTRACT}

Background: ERBB2 (HER2) is a driver gene identified in non-small cell lung cancer (NSCLC). The prevalence, clinicopathology, genetic variability and treatment of HER2-positive NSCLC in Chinese population are unclear.

Patients and Methods: Eight hundred and fifty-nine patients with pathologically confirmed NSCLC were screened for HER2 mutations using Sanger sequencing. Nextgeneration sequencing (NGS) was performed in positive cases. HER2 amplification was detected with FISH. Overall survival (OS) was evaluated using Kaplan-Meier methods and compared with log-rank tests.

Results: Twenty-one cases carrying HER2 mutations were identified with a prevalence of $\mathbf{2 . 4 \%}$. HER2 mutations were more frequently encountered in females, non-smokers and adenocarcinoma. NGS was performed in 19 out of 21 patients, The results showed 16 cases with additional genetic aberrations, most commonly associated with TP53 $(n=6)$, followed by EGFR $(n=3), \operatorname{NF1}(n=3), \operatorname{KRAS}(n=2)$ and other mutations. One patient harbored HER2 amplification. Four patients with stage IV received afatinib treatment, and three showed stable disease with a median progression-free survival of 4 months and one patient was diagnosed with progressive disease.

Conclusion: HER2 mutations represent a distinct subset of NSCLC. NGS showed that HER2 mutations commonly co-existed with other driver genes. Afatinib treatment displayed moderate efficacy in patients with HER2 mutations.

\section{INTRODUCTION}

Lung cancer is one of the leading causes of cancerrelated death worldwide [1]. Testing for driver genes in patients with non-small cell lung cancer (NSCLC) is the new standard of clinical decision-making [2-3]. Targeted therapies focusing on driver genes increase the quality of life and prolong survival [4-7].

ERBB2 (HER2) is expressed in solid carcinomas including cancers of the breast, stomach, lung and pancreas [8]. Preclinical and clinical studies have confirmed that HER2 is a driver gene in NSCLC [912]. Three principal mechanisms of HER2 alteration include: protein overexpression, gene amplification and gene mutations [13]. In NSCLC, HER2 mutations were identified to represent a distinct subset of driver genes that usually excluded with other common driver genes like EGFR,KRAS and ALK , based on published studies. [14$16]$.

Despite the relative rarity of HER2 mutations, several studies have reported HER2 mutations in NSCLC [17-21]. However, most studies focused on the prevalence and clinical characteristics. The prognosis, treatment and especially genetic variability of HER2 have not yet to be well investigated.

In the present study, we investigated the frequency of HER 2 mutations in a large cohort of Chinese NSCLC, along with the treatment and prognosis. We determined genetic variability with next-generation sequencing. 
Table 1: Clinical characteristics comparison in patients with and without HER2 mutations

\begin{tabular}{|l|c|c|c|}
\hline Characteristics & HER2 positive & HER2 negative & $\boldsymbol{P}$ \\
\hline Gender & & & \\
\hline Male & 7 & 493 & 0.019 \\
\hline Female & 14 & 345 & \\
\hline Age & & & 0.198 \\
\hline$<60$ & 10 & 514 & \\
\hline$\geq 60$ & 11 & 322 & \\
\hline Smoking status & & & 0.004 \\
\hline Never & 17 & 412 & \\
\hline Former/current & 4 & 426 & \\
\hline Histology & & & 0.095 \\
\hline Adenocarcinoma & 19 & 624 & \\
\hline Squamous cell cancer & 2 & 214 & \\
\hline Stage at diagnosis & & & 0.825 \\
\hline I-IIIA & 14 & 539 & \\
\hline IIIB/IV & 7 & 299 & \\
\hline
\end{tabular}

\section{RESULTS}

\section{Patient characteristics}

Totally, eight hundred and fifty-nine patients with pathologically confirmed NSCLC were screened for HER2 mutations. Twenty-one patients were identified carrying HER2 mutations. Among the 21 patients, 14 were females and seven of males. The patients' median age was 60 years (range, 39-70). Four patients were ever or current smokers and 17 without smoking history. The majority of patients presented with adenocarcinoma ( $n=$ 19), and the remaining two patients were diagnosed with squamous cell carcinoma. All the stages were represented at diagnosis: four patients with stage I, three patients with stage II, seven patients with stage III, and seven patients with stage IV disease.HER2 mutations occurred at a significantly higher frequency in female, never-smokers with adenocarcinoma when compared with HER2 negative patients. The clinicopathological characteristics of present study are listed in Table 1.

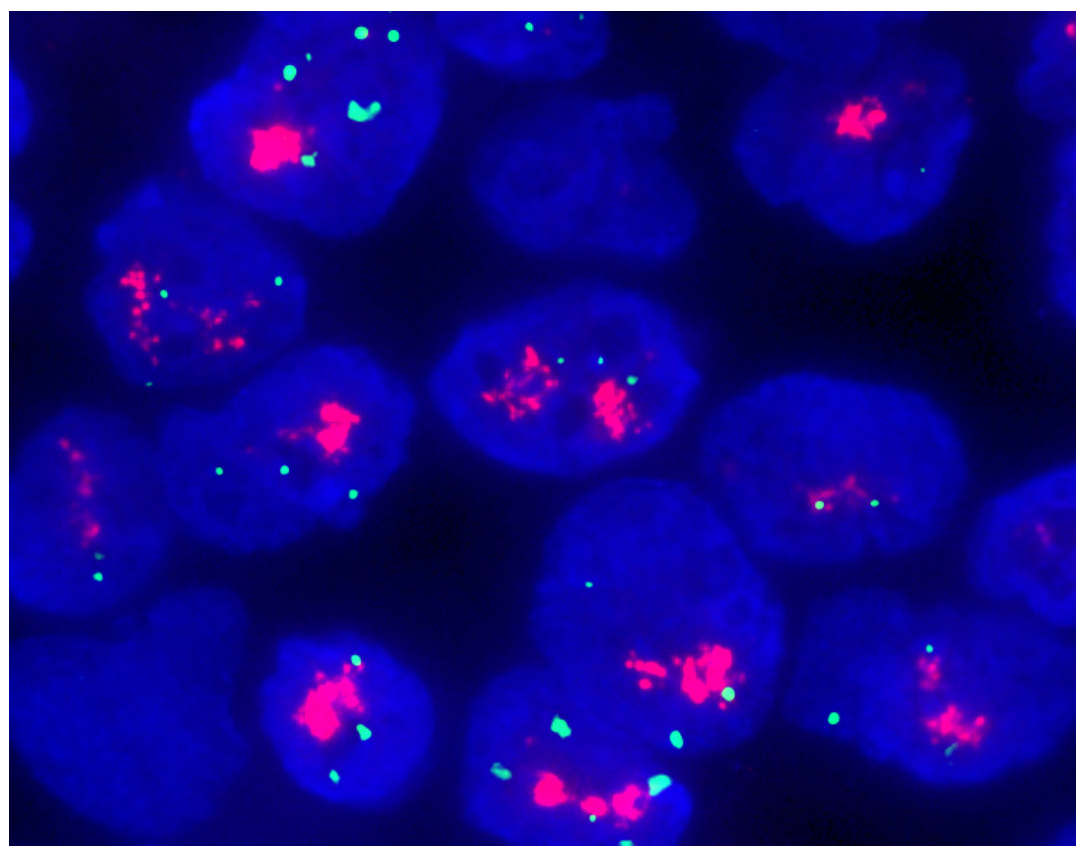

Figure 1: HER2 amplification in a patient (NO. 12) with a HER/CEP17 ratio of 7.3 by FISH detection. 
Table 2: Clinical characteristics of patients carrying a HER2 mutation in their tumors $(n=21)$

\begin{tabular}{|c|c|c|c|c|c|c|c|c|c|c|c|}
\hline Case & $\begin{array}{l}\text { Gender/ } \\
\text { age }\end{array}$ & Histology subtype & $\begin{array}{l}\text { HER2 } \\
\text { subtype }\end{array}$ & Co-current genes & $\begin{array}{l}\text { First-line } \\
\text { chemotherapy }\end{array}$ & efficacy & $\begin{array}{l}\text { PFS/ } \\
\text { months }\end{array}$ & $\begin{array}{l}\text { EGFR- } \\
\text { TKI }\end{array}$ & efficacy & $\begin{array}{l}\text { PFS/ } \\
\text { month }\end{array}$ & OS/month \\
\hline 1 & $\mathrm{M} / 69$ & Enteric & VC $777-778$ ins & KRAS & - & - & - & - & - & - & $60+$ \\
\hline 2 & $\mathrm{~F} / 41$ & Lepidic pred & YVMA 776-779 ins & No detection & Pemetrexed/carboplatin & PR & 5.5 & - & - & - & $55+$ \\
\hline 3 & $\mathrm{~F} / 58$ & Solid pred & GSP 781-783ins & TP53 & Gemcitabine/cisplatin & SD & 4.6 & Afatinib & SD & 5.5 & 21 \\
\hline 4 & $\mathrm{~F} / 65$ & Solid pred & YVMA 776-779 ins & EGFR & Pemetrexed/carboplatin & PD & 1.2 & Icotinib & PD & 1.2 & 12.5 \\
\hline 5 & $\mathrm{~F} / 58$ & Papillary pred & YVMA 776-779 ins & TP53 & - & - & - & - & - & - & $56+$ \\
\hline 6 & $\mathrm{M} / 65$ & Papillary pred & YVMA 776-779 ins & NF1+TP53 & Gemcitabine/cisplatin & SD & 4.8 & Icotinib & PD & 1.1 & 18 \\
\hline 7 & $\mathrm{~F} / 55$ & Papillary pred & YVMA 776-779 ins & No detection & - & - & - & - & -- & - & 45 \\
\hline 8 & $\mathrm{~F} / 50$ & Micropapillary pred & YVMA 776-779 ins & EGFR & Pemetrexed/cisplatin & PR & 5.0 & Icotinib & PR & 14.4 & 17.7 \\
\hline 9 & $\mathrm{M} / 39$ & AIS & YVMA 776-779 ins & - & - & - & - & - & - & - & 39 \\
\hline 10 & $\mathrm{~F} / 70$ & Papillary pred & YVMA 776-779 ins & - & Pemetrexed/carboplatin & SD & 2.8 & Afatinib & SD & 3.5 & 14.5 \\
\hline 11 & $\mathrm{~F} / 55$ & Papillary pred & YVMA 776-779 ins & ROS1 & - & - & - & - & - & - & $48+$ \\
\hline 12 & $\mathrm{~F} / 41$ & Solid pred & YVMA 776-779 ins & HER2 amplification & paclitaxel/carboplatin & SD & 4.6 & - & - & - & $58+$ \\
\hline 13 & $\mathrm{~F} / 69$ & Acinar pred & YVMA 776-779 ins & EGFR+TP53 & Gemcitabine/cisplatin & PD & 1.0 & Icotinib & PD & 1.0 & 6.5 \\
\hline 14 & $\mathrm{M} / 62$ & Minimally invasive & YVMA 776-779 ins & KRAS & Gemcitabine/cisplatin & SD & 4.2 & Icotinib & PD & 1.2 & 12 \\
\hline 15 & $\mathrm{~F} / 54$ & Acinar pred & YVMA 776-779 ins & BRCA1 & Gemcitabine/cisplatin & PR & 8.5 & Gefitinib & PD & 2.0 & 24 \\
\hline 16 & $\mathrm{~F} / 64$ & Papillary pred & YVMA 776-779 ins & CDKN2A+ARID1A & - & - & - & - & - & - & $58+$ \\
\hline 17 & $\mathrm{~F} / 63$ & Papillary pred & YVMA 776-779 ins & $\mathrm{NF} 1+\mathrm{DDR} 2+\mathrm{TP} 53$ & Gemcitabine/cisplatin & PR & 10.0 & - & - & - & 45 \\
\hline 18 & $\mathrm{M} / 60$ & Micropapillary pred & YVMA 776-779 ins & - & - & - & - & Afatinib & SD & 4.0 & 45 \\
\hline 19 & $\mathrm{~F} / 55$ & Squamous cell & YVMA 776-779 ins & NF1+TP53 & Gemcitabine/carboplatin & SD & 2.2 & - & - & - & $36+$ \\
\hline 20 & $\mathrm{M} / 64$ & Squamous cell & YVMA 776-779 ins & EXT1,SMARCA4 & Gemcitabine/cisplatin & PR & 5.0 & Erlotinib & SD & 2.2 & 54 \\
\hline 21 & $\mathrm{M} / 66$ & Papillary pred & YVMA 776-779 ins & KRAS & Docetaxel/platinum & PD & 1.0 & Afatinib & PD & 1.0 & 13 \\
\hline
\end{tabular}

Abbreviations:Pred: predominant; Adenocarcinoma in situ:AIS;Stable disease:SD;Progression disease:PD;partial response: PR.

Table 3: Clinical efficacy of afatinib in HER2 mutation NSCLC patients

\begin{tabular}{|l|l|l|l|l|l|}
\hline Author & Number & Male/female & Efficacy & PFS & OS \\
\hline Mazie`res et al.[23] & 3 & $\mathrm{NP}$ & SD:2/PR:1 & NP & NP \\
\hline De Grève et al.[24] & 3 & $0 / 3$ & SD:2/PR:1 & $4 \mathrm{M}$ & $14 \mathrm{M}$ \\
\hline Current study & 4 & $2 / 2$ & SD:3/PD:1 & $3.5 \mathrm{M}$ & $17.75 \mathrm{M}$ \\
\hline
\end{tabular}

Abbreviations: NP:not reported; SD: stable disease;PR:partial response;PD:progression disease;M:month

\section{Gene analysis}

All the mutations were in-frame insertions of exons 20 and 19 resulting in duplication of amino acids YVMA associated with codon YVMA 776-779 ins and two other codons (one with VC 777-778 ins and the other with GSP 781-783ins). HER2 amplification was detected in one patient (No.12) with HER/CEP17 of 7.3 by FISH (Table 2 and Figure 1).

The tumors of 19 patients were further analyzed by NGS. Concurrent driver genes were detected in 16 patients: six carrying TP53 mutation, three harboring $E G F R$ mutations(two with exon19 delete and one with exon 21 L858R mutation), three carrying NF1 mutations, and two patients with KRAS mutation. Additional gene aberrations are listed in Table 2 .

\section{Treatment}

Among the 859 patients, 511 had a postoperative onset of recurrence or metastasis and 423 died. Of the 21 patients with HER2-positve, fourteen with advanced stage or recurrent NSCLC received palliative chemotherapy or targeted treatment. Eleven patients were treated with EGFR-TKIs including seven with first-generation agents (erlotinib, gefitinib or icotinib) and four with the secondgeneration inhibitor (afatinib). In the seven patients treated with first-generation EGFR-TKIs, the median PFS 
was 2.0 months. Notably, three patients were treated for concurrent HER2 and EGFR mutations, and only one patient responded to first-generation EGFR-TKI treatment.

Four patients received afatinib monotherapy. Disease was controlled in three of these patients with a median progression-free survival of 4.0 months. One patient showed progressive disease with afatinib treatment.

All of the 14 patients received first-line chemotherapy. The median PFS of first-line treatment was 4.6 months. No PFS difference of first-line chemotherapy existed between

Patients with and without HER2 mutations in their tumors (4.6 vs.5.2 months, $P=0.45)$.

\section{Survival analysis}

Of the 859 patients, 62 were lost to follow-up. None of the 21 patients with a HER2 mutations in their tumor were lost to follow-up. No differences in age, histology or stage were seen between HER2-positive and HER2negative patients. The median overall survival in the current study was 49.1 months. HER2-negative patients presented with a tendency for longer survival than HER2positive patients (49.3 months $v s .45 .0$ months, $P=0.150$ ) (Figure 2).

\section{DISCUSSION}

We evaluated the prevalence, prognosis and genetic variability of HER2 mutations in a large cohort of lung cancers in Asian population. Our results demonstrated that patients with a HER2 mutation in their tumor were responsive to HER2 inhibitor treatment. HER2 mutations frequently encountered with other driver genes, which may influence therapeutic decision-making.

The prevalence of HER2 mutations has been reported previously to range from $1 \%$ to $6 \%$ in NSCLC [16-22]. Wang and colleagues reported 1943 NSCLC and found a HER 2 mutation rate of $1.3 \%$. Eleven of 204 NSCLC patients harbored HER2 mutations by Shan et al. study [20]. Based on previous studies, the presence of HER2 mutations was associated with female, never smokers and lung adenocarcinoma. In the present study, HER 2 mutations were associated with never-smoker status and female patients, which is consistent with previous studies [16-22]. Notably, two patients with HER2 mutations were confirmed with squamous cell carcinoma in our cohort. To our knowledge, our report of the two cases involving squamous cell carcinoma with a HER2 mutation is the first of its kind.

In previous studies, HER2 amplification was not routinely detected. Hence, no data of concurrent amplification and mutation were reported. In the present study, HER2 amplification was detected in one patient using FISH. The results suggested that the two molecular alterations were not commonly associated.

Due to the rarity of HER2 mutations in NSCLC, no study investigated genes co-existing with these mutations. In current report, NGS was used to detect the HER2 mutation and concurrent genes. We found that

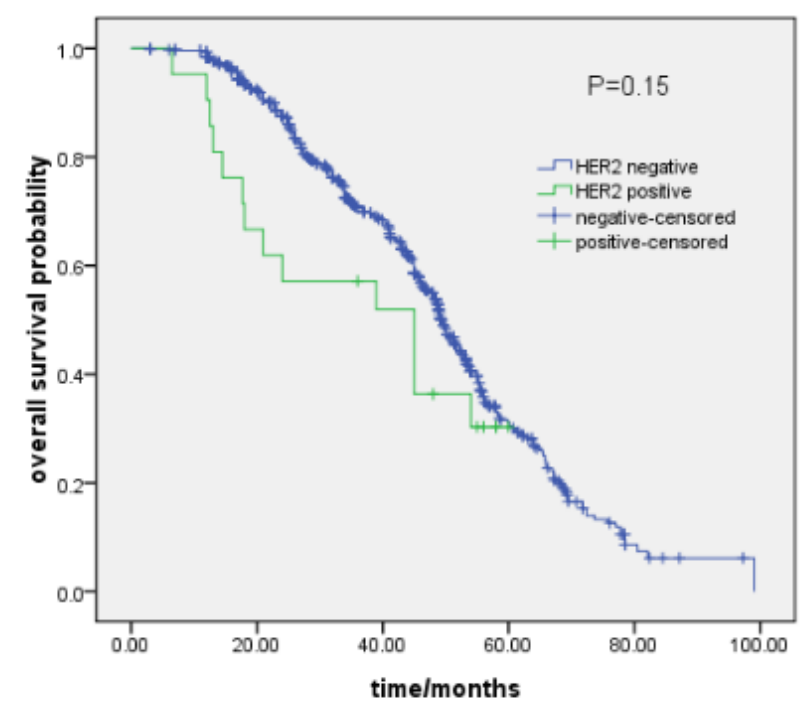

Figure 2: Survival analysis: HER2-positive $v$ s. HER2-negative patients (49.3 months $v s .45 .0$ months, $P=0.150)$. 
16 of 19 patients with a HER2 mutation in their tumors carried concurrent genes. Notably, three of our concurrent HER2 and EGFR-positive patients were treated with firstgeneration EGFR-TKI inhibitors (two with exon19 delete and one with exon21 L858R mutation), and two presented with progressive disease. The limited number of patients in the current study suggested the poor efficacy of firstgeneration EGFR-TKIs against HER2 mutations. In these patients, second-generation EGFR-TKIs such as afatinib and dacomtinib may be a better option.

Evidence supporting HER2-targeted therapy in NSCLC is still scarce. In the largest published series, Mazieres et al. reported that 16 patients with HER2 mutation were treated resulting in a median PFS of 5.1 months [23]. The anti-HER2 therapies included trastuzumab, afatinib and lapatinib. Interestingly, three patients were treated with afatinib resulting in $100 \%$ disease control. De Grève et al. reported that three NSCLC patients with HER2 mutation treated with afatinib showed objective response [24]. We report disease control in three out of four patients with HER2 mutations treated with afatinib. Based on these two studies and our data (Table 3 ), we conclude that afatinib monotherapy is appropriate for patients with HER 2 mutations. In addition, recent data have demonstrated that afatinib plus paclitaxel may be particularly more effective than single afatinib treatment, hence, combinitaion afatinib and chemotherapy may be a better option in HER2-positive NSCLC patients [25].

Due to the rarity of the molecular subtypes, the survival of HER2 mutations in NSCLC was not well studied. In one study by Tomizawa et al., a tendency of shorter overall survival in HER2 mutations was reported compared with HER2-negative cases [26]. In another cohort, the overall survival of HER2 mutations did not differ significantly from other cohorts carrying $E G F R$, $A L K, K R A S$, and $B R A F$ mutations [10]. In the present cohort, no survival differences were found in patients with and without HER2 mutations. However, there was a trend toward longer survival in HER2-negative patients. The results may due to the imbalance in treatment after recurrence or metastasis.

Our study limitations are related to its retrospective design with only 21 patients with HER 2 mutation. Further, only four patients were treated with afatinib, suggesting caution in interpreting afatinib efficacy in HER2 mutants.

Our report suggests that the disease with HER2 mutations represents a subset of NSCLC. We suggest that HER2 mutations are not exclusive to other genetic aberrations detected by NGS. We also found that afatinib is a potentially novel treatment option for this subgroup of patients.

\section{MATERIALS AND METHODS}

\section{Patient selection}

A total of 859 patients with pathologically-confirmed NSCLC at Zhejiang Cancer Hospital from January 2008 through December 2014, were retrospectively enrolled in this study. The inclusion criteria were: pathologically confirmed diagnosis of NSCLC, availability of samples for HER2 analysis, and absence of chemotherapy or radiotherapy before pathological diagnosis. Histology was based on the 2004 World Health Organization classification. Lung cancer staging was performed in all patients according to the $7^{\text {th }}$ TNM classification. This study was approved by the Review Board of the Zhejiang Cancer Hospital, Zhejiang, China.

\section{HER2 mutation analysis}

A microscopy was used to ensure the tumor tissues analyzed had more than 20\% tumor contents. Genomic DNA was extracted using the QIAamp DNA Tissue kit (Qiagen,Germany) following the manufacturer's standard protocol. HER2 exon 20 mutation analysis was carried out using Sanger sequencing. Briefly, the entire coding region of HER 2 exon 20 was amplified using the forward primer, 5'-GCCATGGCTGTGGTTTGTGATGG-3' and reverse primer,5'-ATCCTAGCCCCTTGTGGACATAGG-3.The

Refseq accession number for HER2 gene analyzed in this study is NM_001289937.

\section{HER2 FISH}

The Vysis PathVysion HER2 DNA Probe Kit (Abbott Laboratories) was used for HER amplification according to the standard manufacturer's protocol. At least 40 cells were analyzed in each case by two pathologists and classified according to the $2013 \mathrm{ASCO} /$ CAP evaluation systems for HER2 amplification in breast cancer: $H E R 2 / C E P 17$ ratio $\geq 2.0$ or $<2.0$, average $H E R 2$ copy number $\geq 6.0$ signals/cell.

\section{NGS detection}

The HER2 samples were subjected to targeted next generation sequencing (NGS). DNA was extracted by digestion in proteinase $\mathrm{K}$ buffer, followed by purification with DNeasy Tissue kit (Qiagen) with some modifications. The 416 cancer-related genes were captured using a 5'-biotinylated probe. Deep sequencing was performed with IlluminaHiSeq4000 using PE75 V1 Kit (Illumina, San Diego, USA). Cluster generation and sequencing was performed according to the manufacturer's protocol. 
Results were visualized in the Integrative Genomics Viewer (IGV) and manually analyzed. A 1\% cutoff for variant calls was used. The median sequencing coverage was $>800$. A comprehensive protocol of NGS is provided as a supplementary file.

\section{Efficacy evaluation}

Tumor evaluation during afatinib treatment was performed every four to eight weeks, or earlier if significant signs of progression were observed. Objective tumor responses were evaluated based on the Response Evaluation Criteria in Solid Tumors (RECIST 1.1).

\section{Statistical analysis}

The relationship between HER2 mutation and clinicopathological variables was analyzed using the chisquare test. Survival curves were calculated using the Kaplan-Meier method starting with NSCLC diagnosis until death or last follow-up. Progression-free survival (PFS) of EGFR-TKI was defined as the time from therapy to documented progression or death due to any cause. Statistical analysis was performed with the SPSS 16 software (Chicago, IL, US). $P<0.05$ was considered statistically significant. The median follow-up period was 55 months (5-75) and the last follow-up was conducted on July $31,2015$.

\section{CONFLICTS OF INTEREST}

There is no conflict of interest.

\section{REFERENCES}

1. Siegel R, Ma J, Zou Z, Jemal A. Cancer statistics. CA Cancer J Clin. 2014; 64:9-29.

2. Kris MG, Johnson BE, Berry LD, Kwiatkowski DJ, Iafrate AJ, Wistuba II, Varella-Garcia M, Franklin WA, Aronson SL, Su PF, Shyr Y, Camidge DR, Sequist LV, et al. Using multiplexed assays of oncogenic drivers in lung cancers to select targeted drugs. JAMA.2014;311:1998-2006.

3. Sholl LM, Aisner DL, Varella-Garcia M, Berry LD, DiasSantagata D, Wistuba II, Chen H, Fujimoto J, Kugler K, Franklin WA,Iafrate AJ, Ladanyi M, Kris MG, Johnson BE, Bunn PA, Minna JD, Kwiatkowski DJ;Multiinstitutional Oncogenic Driver Mutation Analysis in Lung Adenocarcinoma The Lung Cancer Mutation Consortium Experience.J Thorac Oncol. 2015;10(5):768-777.

4. Kwak EL, Bang YJ, Camidge DR, Shaw AT, Solomon B, Maki RG, Ou SH, Dezube BJ, Janne PA, Costa DB, Varella-Garcia M, Kim WH, Lynch TJ, et al. Anaplastic lymphoma kinase inhibition in non-small-cell lung cancer. The New England journal of medicine. 2010; 363:1693-
1703.

5. Shaw AT, Kim DW, Mehra R, Tan DS, Felip E, Chow LQ, Camidge DR, Vansteenkiste J, Sharma S, De Pas T, Riely GJ, Solomon BJ, Wolf J, et al. Ceritinib in ALK-rearranged non-small-cell lung cancer. N Engl J Med. 2014; 370:11891197.

6. Rosell R, Carcereny E, Gervais R, Vergnenegre A,Massuti B, Felip E, Palmero R, Garcia-Gomez R,Pallares C, Sanchez JM, Porta R, Cobo M, Garrido P, et al. Erlotinib versus standard chemotherapy as first-line treatment for European patients with advanced EGFR mutation-positive non-small-cell lung cancer (EURTAC): a multicentre, openlabel, randomised phase 3 trial. The lancet oncology. 2012; 13:239-246.

7. Mok TS, Wu YL, Thongprasert S, Yang CH, Chu DT, Saijo $\mathrm{N}$, Sunpaweravong $\mathrm{P}$, Han B, Margono B, Ichinose Y,Nishiwaki Y, Ohe Y, Yang JJ, et al. Gefitinib or carboplatin-paclitaxel in pulmonary adenocarcinoma. The New England journal of medicine. 2009; 361:947-957.

8. Scholl S, Beuzeboc P, Pouillart P. Targeting HER2 in other tumor types. Ann Oncol. 2001;12(Suppl. 1):S81-7.

9. Wang SE, Narasanna A, Perez-Torres M, Xiang B, Wu FY, Yang S, Carpenter G, Gazdar AF, Muthuswamy SK, Arteaga CL.HER2 kinase domain mutation results in constitutive phosphorylation and activation of HER2 and EGFR and resistance to EGFR tyrosine kinase inhibitors. Cancer Cell. 2006;10:25-38.

10. Arcila ME, Chaft JE, Nafa K, Roy-Chowdhuri S, Lau C, Zaidinski M, Paik PK, Zakowski MF, Kris MG, Ladanyi M.Prevalence, clinicopathologic associations, and molecular spectrum of ERBB2 (HER2) tyrosine kinase mutations in lung adenocarcinomas. Clin Cancer Res. 2012;18:4910-4918.

11. Buttitta F, Barassi F, Fresu G, Felicioni L, Chella A, Paolizzi D, Lattanzio G, Salvatore S, Camplese PP, Rosini S, Iarussi T,Mucilli F, Sacco R, Mezzetti A, Marchetti A.Mutational analysis of the HER2 gene in lung tumors from Caucasian patients: mutations are mainly present in adenocarcinomas with bronchioloalveolar features. Int $\mathbf{J}$ Cancer. 2006;119:2586-2591.

12. Li C, Fang R, Sun Y, Han X, Li F, Gao B, Iafrate AJ, Liu XY, Pao W, Chen H, Ji H. Spectrum of oncogenic driver mutations in lung adenocarcinomas from East Asian never smokers. PLoS One. 2011;6:e28204.

13. Mar N, Vredenburgh JJ, Wasser JS.Targeting HER2 in the treatment of non-small cell lung cancer.Lung Cancer. 2015;87:220-225.

14. Wu C, Zhao C, Yang Y, He Y, Hou L, Li X, Gao G, Shi J, Ren S, Chu H, Zhou C, Zhang J, Schmid-Bindert G.High Discrepancy of Driver Mutations in Patients with NSCLC and Synchronous Multiple Lung Ground-Glass Nodules. J Thorac Oncol. 2015;10:778-783.

15. Zhang Y, Sun Y, Pan Y, Li C, Shen L, Li Y, Luo X, Ye T, Wang R, Hu H, Li H, Wang L, Pao W, Chen H.Frequency 
of driver mutations in lung adenocarcinoma from female never-smokers varies with histologic subtypes and age at diagnosis. Clin Cancer Res. 2012;18:1947-1953.

16. Sun Y, Ren Y, Fang Z, Li C, Fang R, Gao B, Han X, Tian W, Pao W, Chen H, Ji H.Lung adenocarcinoma from East Asian never-smokers is a disease largely defined by targetable oncogenic mutant kinases.J Clin Oncol.2010;28:4616-4620.

17. Li H, Pan Y, Li Y, Li C, Wang R, Hu H, Zhang Y, Ye T, Wang L, Shen L, Sun Y, Chen H. Frequency of well-identified oncogenic driver mutations in lung adenocarcinoma of smokers varies with histological subtypes and graduated smoking dose.Lung Cancer. 2013 ;79:8-13.

18. Ren S, Kuang P, Zheng L, Su C, Li J, Li B, Chen X, Wang Y, KimCurran V, Liu L, Hu Q, Zhang J, Tang L, Zhou C.Analysis of driver mutations in female non-smoker Asian patients with pulmonary adenocarcinoma.Cell Biochem Biophys. $2012 ; 64: 155-60$.

19. Peters S, Zimmermann S. Targeted therapy in NSCLC driven by HER2 insertions. Transl Lung Cancer Res .2014 ;3:84-88.

20. Shan L, Qiu T, Ling Y, Guo L, Zheng B, Wang B, Li W, Li L, Ying J.Prevalence and Clinicopathological Characteristics of HER2 and BRAF Mutation in Chinese Patients with Lung Adenocarcinoma.PLoS One. 2015 ;10(6):e0130447.

21. Wang R, Zhang Y, Pan Y, Li Y, Hu H, Cai D, Li H, Ye T, Luo X, Zhang Y, Li B, Shen L, Sun Y, Chen H.Comprehensive investigation of oncogenic driver mutations in Chinese non-small cell lung cancer patients.Oncotarget. 2015 ;6:34300-8. doi: 10.18632/ oncotarget.5549.
22. Li BT, Ross DS, Aisner DL, Chaft JE, Hsu M, Kako SL, Kris MG, Varella-Garcia M, Arcila ME.HER2 Amplification and HER2 Mutation Are Distinct Molecular Targets in Lung Cancers.J Thorac Oncol. 2016 ;11:414-9.

23. Mazières J, Peters S, Lepage B, Cortot AB, Barlesi F, Beau-Faller M, Besse B, Blons H, Mansuet-Lupo A, Urban T, Moro-Sibilot D, Dansin E, Chouaid C, Wislez M, Diebold J, Felip E, Rouquette I, Milia JD, Gautschi O.Lung Cancer That Harbors an HER2 Mutation: Epidemiologic Characteristics and Therapeutic Perspectives.J Clin Oncol. 2013;31:1997-2003.

24. De Grève J, Teugels E, Geers C, Decoster L, Galdermans D, De Mey J, Everaert H, Umelo I, In't Veld P, Schallier D.Clinical activity of afatinib (BIBW 2992) in patients with lung adenocarcinoma with mutations in the kinase domain of HER2/neu. Lung Cancer. 2012;76:123-127.

25. Schuler M, Yang JC, Park K, Kim JH, Bennouna J, Chen YM, Chouaid C, De Marinis F, Feng JF, Grossi F, Kim DW, Liu X, Lu S, Strausz J, Vinnyk Y, Wiewrodt R, Zhou C, Wang B, Chand VK, Planchard D.Afatinib beyond progression in patients with non-small-cell lung cancer following chemotherapy, erlotinib/gefitinib and afatinib: phase III randomized LUX-Lung 5 trial.Ann Oncol. 2016;27:417-23.

26. Tomizawa K, Suda K, Onozato R, Kosaka T, Endoh H, Sekido Y, Shigematsu H, Kuwano H, Yatabe Y, Mitsudomi T. Prognostic and predictive implications of HER2/ ERBB2/neu gene mutations in lung cancers. Lung Cancer. 2011;74:139-144. 